\title{
FGFR2-related pathogenesis and FGFR2-targeted therapeutics (Review)
}

\author{
YURIKO KATOH $^{1}$ and MASARU KATOH ${ }^{2}$ \\ ${ }^{1}$ M\&M Medical BioInformatics, Hongo 113-0033; ${ }^{2}$ Genetics and Cell Biology Section, \\ National Cancer Center, Tokyo 104-0045, Japan
}

Received December 5, 2008; Accepted December 31, 2008

DOI: 10.3892/ijmm_00000132

\begin{abstract}
FGFR2 gene at human chromosome 10q26 encodes FGFR2b and FGFR2c isoforms functioning as FGF receptors with distinct expression domain and ligand specificity. FGFR2 plays oncogenic and anti-oncogenic roles in a contextdependent manner. Single nucleotide polymorphisms (SNPs) within intron 2 of FGFR2 gene are associated with breast cancer through allelic FGFR2 upregulation. Missense mutations or copy number gains of FGFR2 gene occur in breast cancer and gastric cancer to activate FGFR2 signaling. Aberrant FGFR2 signaling activation induces proliferation and survival of tumor cells. The class switch from FGFR2b to FGFR2c occurs during progression of prostate cancer and bladder cancer because of spliceosome dysregulation. In addition, epidermal $F g$ fr $2 b$ knockout mice show increased sensitivity to chemical carcinogenesis partly due to the failure of Nfe212 (Nrf2)-mediated detoxification of reactive oxygen species (ROS). Loss of FGFR2b signaling induces epithelialto-mesenchymal transition (EMT) and unruly ROS. FGFR2 signaling dysregulation due to the accumulation of epigenetic modifications and genetic alterations during chronic inflammation, smoking, increased caloric uptake, and decreased exercise leads to carcinogenesis. PD173074, SU5402, AZD2171, and Ki23057 are small-molecule FGFR inhibitors. Human antibody, peptide mimetic, RNA aptamer, siRNA, and synthetic microRNA (miRNA) are emerging technologies to be applied for cancer therapeutics targeted to FGFR2. Because novel sequence technology and peta-scale supercomputer are opening up the sequence era following the genome era, personalized medicine prescribing targeted drugs based on germline and/or somatic genomic information is coming reality. Application of FGFR2 inhibitors for cancer treatment in patients with FGFR2
\end{abstract}

Correspondence to: Dr Masaru Katoh, Genetics and Cell Biology Section, National Cancer Center, 5-1-1 Tsukiji, Chuo Ward, Tokyo 104-0045, Japan

E-mail: mkatoh-kkr@umin.ac.jp

Key words: KEAP1, ubiquitylation, chemoradiotherapy, esophageal cancer, FGF7, WNT, Hedgehog, invasion, metastasis, peritoneal dissemination mutation or gene amplification is beneficial; however, that for cancer prevention in people with $F G F R 2$ risk allele might be disadvantageous due to the impediment of a cytoprotective mechanism against oxidative stress.
Contents
1. Introduction
2. Oncogenic FGFR2 in human cancer
3. Anti-oncogenic FGFR2b
4. FGFR2 in non-cancerous disorders
5. Small-molecule FGFR inhibitors
6. Other therapeutics targeted to FGFR2
7. Conclusion and perspectives

\section{Introduction}

FGFR2 gene at human chromosome 10q26 encodes FGFR2b and FGFR2c isoforms due to alternative splicing (1-4). FGFR2b and FGFR2c function as fibroblast growth factor (FGF) receptors transducing FGF signals to RAS-ERK and PI3K-AKT signaling cascades through FRS2, and also to DAG-PKC and IP3-Calmodulin signaling cascades through PLC $\gamma(5,6)$. FGFR2b and FGFR2c with extracellular immunoglobulin-like domains and cytoplasmic tyrosine kinase domain are almost identical except the latter half of the third immunoglobulin-like domain.

Epithelial cells moving as a sheet en block are tightly held together with uniform neighboring cells, while mesenchymal cells moving individually are loosely connected with diverse neighboring cells (7-9). FGFR2b on epithelial cells is a high affinity receptor for FGF1, FGF3, FGF7, FGF10 and FGF22 $(10,11)$. FGFR2c on mesenchymal cells is a high affinity receptor for FGF1, FGF2, FGF4, FGF6, FGF9, FGF16 and FGF20 (10,11). FGFR2b and FGFR2c show distinct expression domain and ligand specificity.

FGF7, FGF10, and FGF22 constitute a subfamily among the FGF family (12-15). FGF7, induced by PDGF, IL-1, IL-1ß or TNF- $\alpha$, is secreted from fibroblast, smooth muscle cells, endothelial cells, skin dermis, and $\gamma \delta \mathrm{T}$ cells to promote tissue repair (16-18). FGF10 is secreted from mesenchymal cells to orchestrate morphogenesis of gastrointestinal tract, respiratory tract, limb, and other organs or tissues $(19,20)$. FGF22 is 
secreted from cerebellar granule cells to regulate synapse formation (21). FGF7, FGF10 and FGF22 transduce signals through FGFR2b on epithelial cells to regulate embryogenesis and adult tissue homeostasis (18).

FGF signaling cascades interact with WNT, Notch, Hedgehog and BMP signaling cascades to constitute the stem cell signaling network $(22,23)$. Dysregulation of the stem cell signaling network caused by the accumulation of epigenetic modifications and genetic alterations due to single nucleotide polymorphism (SNP), chronic inflammation, smoking, increased caloric uptake and decreased exercise leads to carcinogenesis (24). Because dysregulation of FGFR2 signaling is involved in cancer and congenital disorders $(25,26)$, pathogenesis related to FGFR2 will be reviewed at first, and then therapeutics targeted to FGFR will be described with the emphasis on future clinical application.

\section{Oncogenic FGFR2 in human cancer}

Missense mutations of FGFR2 gene occur in endometrial uterus cancer, ovarian cancer, breast cancer, lung cancer and gastric cancer (27-29). FGFR2 mutations around the third immunoglobulin-like domain result in FGFR2 signaling activation due to the creation of autocrine FGF signaling loop, while those within tyrosine kinase domain results in FGFR2 signaling activation due to the release of FGFR2 from autoinhibition as previously reviewed (25). Copy number gains of FGFR2 gene in breast cancer and gastric cancer result in FGFR2 signaling activation due to overexpression of FGFR2 (30,31). In addition, C-terminal deletion of FGFR2 occurs during gene amplification process due to the exclusion of the last exon from FGFR2 amplicon, which results in FGFR2 signaling activation based on the constitutive phosphorylation of FRS2 adaptor molecule (32). Point mutation or gene amplification of $F G F R 2$, inducing aberrant FGFR2 signaling activation, is involved in human carcinogenesis (Fig. 1).

Recently, a variety of cancer-associated SNPs have been identified based on genome-wide association study (GWAS). Eight SNPs (rs35054928, rs2981578, rs2912778, rs2912781, rs35393331, rs10736303, rs7895676, and rs33971856) within intron 2 of FGFR2 gene are associated with increased risk of breast cancer (33-36). Perfect POU (Oct)-binding site is located adjacent to rs35054928 and rs2981578, and putative RUNX-binding site is created on risk allele of rs 2981578 . Putative estrogen receptor (ER)-binding site is created on risk allele of rs10736303. Putative C/EBPß-binding site is lost from risk allele of rs7895676. Breast cancer-associated allele of rs 2981578 is associated with FGFR2 upregulation in the reporter assay (36); however, precise mechanism how FGFR2 upregulation is induced by FGFR2 risk allele spanning the putative enhancer region within intron 2 remains unclear.

Breast cancer-associated allele rs2981578 mentioned above is associated with decreased risk of endometrial uterus cancer (37), and is not associated with risk of epithelial ovarian cancer (38). Because of the diversity of genetic background and carcinogenic scenario, association between FGFR2 SNPs and risks of several types of cancer should be further investigated among several populations in the world (25).
Regulatory SNP in enhancer region

Activating missense mutation

Copy number gain

$\sqrt{ }$

Aberrant FGFR2 signaling activation

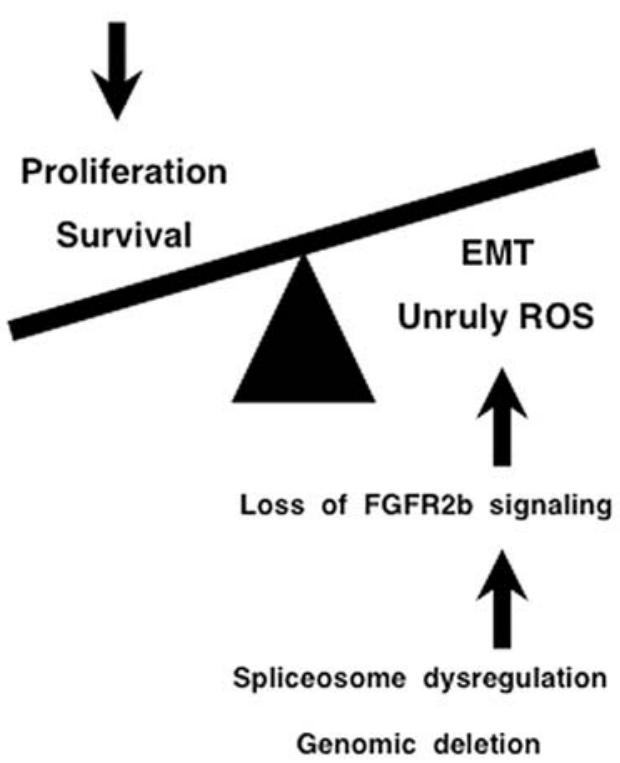

Figure 1. Oncogenic and anti-oncogenic FGFR2.

\section{Anti-oncogenic FGFR2b}

Class switch from FGFR2b to FGFR2c occurs during progression process of prostate cancer and bladder cancer (39), which is accompanied by epithelial-to-mesenchymal transition (EMT) with increased potential for invasion and metastasis $(7-9,40,41)$. Proliferation and tumorigenicity of prostate or bladder cancer cells with decreased FGFR2b expression are significantly suppressed by the transfection of FGFR $2 b$ expression construct $(42,43)$. FGFR2b is anti-oncogenic in prostate cancer and bladder cancer.

Fgf7, Fgf10, and Fgf22 transduce signals through Fgfr2b in skin epidermis (18). Mice with conditional Fgfr2b knockout in skin epidermis show increased occurrence of squamous cell carcinoma (SCC) with oncogenic Hras mutations after DMBA/TPA treatment, indicating that loss of Fgfr2b in skin epidermis results in increased sensitivity to chemical carcinogenesis (44). Fgf7 and Fgf10 are in part secreted from $\gamma \delta \mathrm{T}$ cells within epidermis during wound healing process (45), and mice lacking $\gamma \delta \mathrm{T}$ cells also show enhanced sensitivity to skin carcinogenesis in the DMBA/TPA model (46). Together these facts indicate that inactivation of Fgfr2b signaling promotes mouse skin carcinogenesis (Fig. 1).

$N f e 2 l 2(N r f 2)$ is one of target genes of the Fgfr2b signaling pathway in the skin epidermis (18). $N f e 2 l 2$ gene encodes a basic leucine zipper (bZIP) transcription factor Nfe212 homologous to Nfe2 and Nfe211 (Nrf1). Although Nfe212 is downregulated due to Keap1-mediated ubiquitylation under 
non-stressed condition, Nfe212 is released from Keap1-induced degradation due to structural modification of Keap1 under environmental or endogenous reactive oxygen species (ROS) (47). Stabilized Nfe212 binds to antioxidant (ROS-detoxifying) response element of target genes encoding antioxidant enzymes to catalyze carcinogens to non-carcinogenic chemicals. Because Fgfr2b signaling upregulates Nfe212 involved in cytoprotection (18), loss of Fgfr2b signaling due to deletion or spliceosome dysregulation accelerates carcinogenesis.

\section{FGFR2 in non-cancerous disorders}

Missense activating mutations of FGFR2 gene occur in Crouzon syndrome, Jackson-Weiss syndrome, Apert syndrome, Pfeiffer syndrome, and Beare-Stevenson syndrome, which are congenital skeletal disorders manifested by short-limbed bone dysplasia (craniosynostosis), and other features specific to each syndrome, such as Crouzonoid faces, bone syndactyly, limb abnormalities, and cutis gyrata (25,48-50). Missense mutations of FGFR2 gene induce aberrant FGFR2 signaling activation during skeletal development.

One SNP (rs17101921) located in the 3'-flanking region of FGFR2 gene is claimed to be associated with schizophrenia based on the analyses on 10 cases (51); however, confirmatory study using $>1000$ cases and controls are mandatory to reach the conclusion. Because FGFR2 and WDR 11 genes are clustered around the recombination hot spot at human chromosome 10q26 (4), real causative gene associated with rs17101921 SNP remains unclear. It is noteworthy that Fgf22-Fgfr2b signaling cascade is involved in synapse formation during embryogenesis. Candidate approach to investigate SNPs of genes encoding FGF22-FGFR2b signaling components might be useful to identify novel causative SNPs associated with schizophrenia.

\section{Small-molecule FGFR inhibitors}

Protein kinases with conserved amino-acid sequence share the catalytic domain with similar three-dimensional structure. Small-molecule compounds fitting into the ATP-binding pockets of protein kinases have been developed for cancer therapeutics (52,53). PD173074, SU5402, AZD2171, and Ki23057 are representative small-molecule FGFR inhibitors (Fig. 2).

PD173074 with pyrido[2,3-d]pyrimidine core inhibits FGFR1 with $\mathrm{IC}_{50}$ value of $20 \mathrm{nM}$ (54). PD173074 interacts with L484, V492, A512, K514, E531, M535, I545, V559, V561, Y563, A564, L630, A640, and F642 around the ATPbinding pocket of FGFR1, and inhibits tyrosine kinase activity and autophosphorylation of FGFR1. PD173074 blocks FGF2induced angiogenesis in vivo (54). PD173074 also blocks mitogenesis of tumor cells through G1-arrest mediated by downregulation of Cyclin D1 and Cyclin D2 at the concentration of $2000 \mathrm{nM}$ (55). PD173074 inhibits proliferation and survival of endometrial cancer cells with FGFR2 mutations $(56,57)$.

SU5402 with indolin-2-one core inhibits FGFR1, PDGFRB and VEFGR2 tyrosine kinases with $\mathrm{IC}_{50}$ values of $30 \mathrm{nM}$, $510 \mathrm{nM}$ and $20 \mathrm{nM}$, respectively (58). The indolin-2-one core

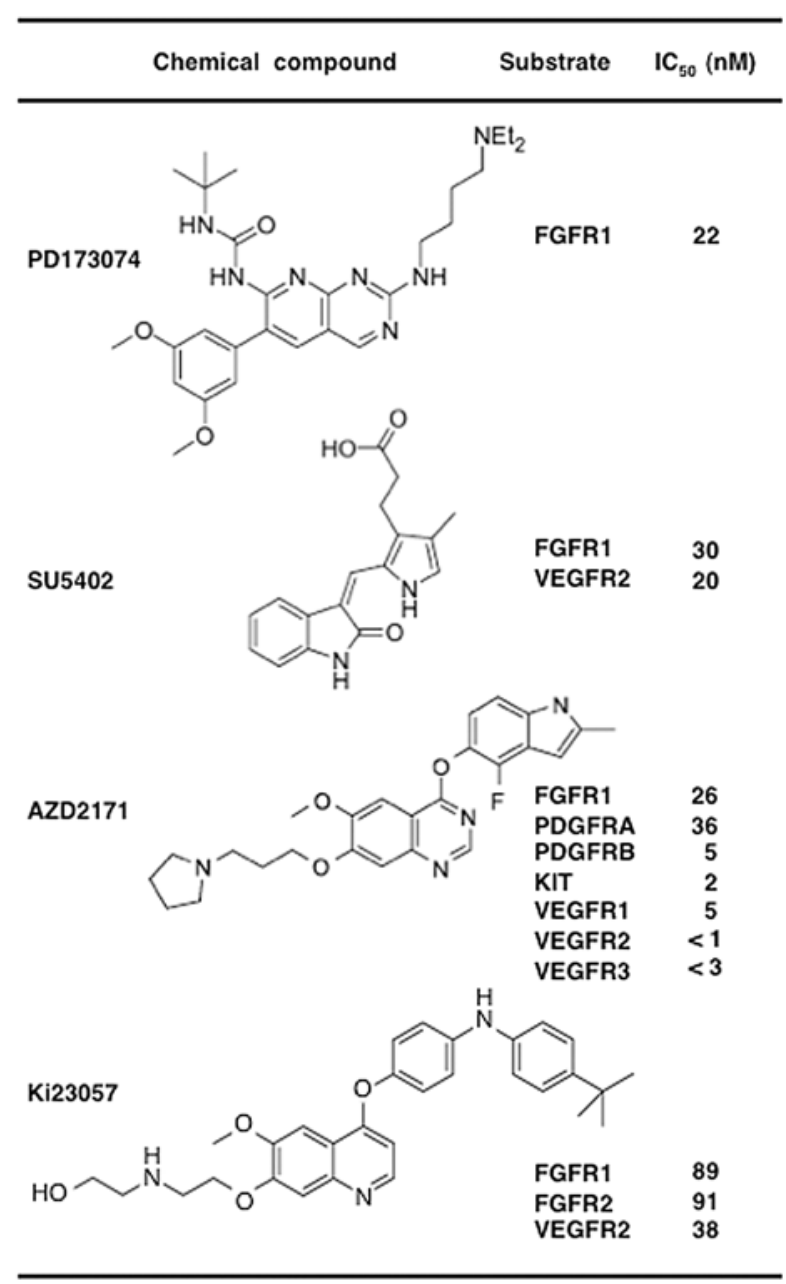

Figure 2. Small-molecule FGFR inhibitors.

of SU5402 interacts with the ATP-binding site of FGFR1 kinase domain, while substituted moieties interact with the hinge region between two lobes of FGFR1 kinase domain (59). Because selectivity of indolin-2-one compounds against receptor tyrosine kinases are determined by substituents extending from the indolin-2-one core, SU5402 is a narrowrange tyrosine kinase inhibitor.

AZD2171 is a broad-range tyrosine kinase inhibitor (60). AZD2171 inhibits FGFR1, PDGFRB and VEFGR2 tyrosine kinases with $\mathrm{IC}_{50}$ values of $26 \mathrm{nM}, 5 \mathrm{nM}$, and $<1 \mathrm{nM}$, respectively. AZD2171 also inhibits other receptor tyrosine kinases, such as PDGFRA, KIT, VEGFR1, and VEGFR3. Oral administration of AZD2171 (1.5 mg/kg/day) significantly inhibits growth of various human tumor cells transplanted into athymic mice (60). AZD2171 inhibits VEGF-induced proliferation of human umbilical vein endothelial cells at concentrations less than $\mathrm{nM}$, and proliferation of human tumor cells at concentrations around mM. AZD2171 inhibits tumor growth in vivo due to indirect effects on endothelial cells rather than direct effects on tumor cells themselves (60).

Ki23057 is also a broad-range tyrosine kinase inhibitor (61). Ki23057 inhibits FGFR1, FGFR2 and VEGF2 tyrosine kinases with $\mathrm{IC}_{50}$ values of $89 \mathrm{nM}, 91 \mathrm{nM}$, and $38 \mathrm{nM}$, respectively. Ki23057 inhibits proliferation of OCUM-2MD3 
and OCUM-8 gastric cancer cells with FGFR2 gene amplification, but not MKN7, MKN45 and MKN74 gastric cancer cells without FGFR2 gene amplification (61). Oral administration of $\mathrm{Ki} 23057$ (25 mg/kg/day) inhibits growth and peritoneal dissemination of OCUM-2MD3 cells. Antitumor effects of Ki23057 are mainly due to FGFR2-RASERK signaling inhibition rather than FGFR2-PI3K-AKT signaling inhibition (61).

\section{Other therapeutics targeted to FGFR2}

Human antibody, peptide mimetic, RNA aptamer, small interfering RNA (siRNA), and synthetic microRNA (miRNA) are emerging technologies to be applied for molecular cancer therapy $(41,62)$.

Peptide mimetic is a promising strategy to develop agonist or antagonist for transmembrane receptors. Dekafins are FGF mimetic peptides associating with FGFR1c or FGFR2b (63). Dekafin1 and Dekafin10 are partial agonists of FGFR1c, and the association between dekafin 1 and FGFR1c is modulated by heparin sulfate moiety. Peptide mimetics functioning as FGFR2 antagonists will be potent lead compounds.

RNA aptamers are short RNA oligonucleotides forming a stable three-dimensional structure for specific tight binding to target protein (64-67). RNA aptamers binding to target proteins are selected from combinatorial libraries by using SELEX method. RNA aptamers targeted to FGFR2 kinase domain or FGFR2-FRS2 interface will be developed as novel FGFR2 signaling inhibitors, while those targeted to FGFR2 extracellular region will be developed as substitutes of human antibody for immunotherapy or tumor-targeted drug delivery.

Fire et al reported RNA repression by using doublestranded RNA in 1998 (68). Elbashir et al reported RNA repression by using siRNA in 2001 (69). Liang et al reported RNA repression by using synthetic miRNA in 2007 (70). siRNA and synthetic miRNA controlling protein expression through target-mRNA degradation or translational repression are promising technologies for cancer therapeutics; however, avoidance of off-target effects and development of tumorspecific delivery system should be addressed before clinical application (62).

\section{Conclusion and perspectives}

FGFR2 plays oncogenic and anti-oncogenic roles in a contextdependent manner (Fig. 1). Because novel sequence technology and peta-scale supercomputer are opening up the sequence era following the genome era (71), personalized medicine prescribing targeted drugs based on germline and/or somatic genomic information is coming reality $(72,73)$. Application of FGFR2 inhibitors for cancer treatment in patients with FGFR2 mutation or gene amplification is beneficial; however, that for cancer prevention in people with FGFR2 risk allele might be disadvantageous due to the impediment of a cytoprotective mechanism against oxidative stress induced by smoking, irradiation, and chronic inflammation.

\section{References}

1. Dionne CA, Crumley G, Bellot F, et al: Cloning and expression of two distinct high-affinity receptors cross-reacting with acidic and basic FGFs. EMBO J 9: 2685-2692, 1990.
2. Miki T, Fleming TP, Bottaro DP, et al: Expression cDNA cloning of the KGF receptor by creation of a transforming autocrine loop. Science 251: 72-75, 1991.

3. Carstens RP, Wagner EJ and Garcia-Blanco MA: An intronic splicing silencer causes skipping of the IIIb exon of $F g f r 2$ through involvement of polypyrimidine tract binding protein. Mol Cell Biol 20: 7388-7400, 2000.

4. Katoh $\mathrm{M}$ and Katoh M: FGFR2 and WDR11 are neighboring oncogene and tumor suppressor gene on human chromosome 10q26. Int J Oncol 22: 1155-1159, 2003.

5. Eswarakumar VP, Lax I and Schlessinger J: Cellular signaling by FGF receptors. Cytokine Growth Factor Rev 16: 139-149, 2005.

6. Dailey L, Ambrosetti D, Mansukhani A and Basilico C: Mechanisms underlying differential responses to FGF signaling. Cytokine Growth Factor Rev 16: 233-247, 2005

7. Thiery JP: Epithelial-mesenchymal transitions in tumour progression. Nat Rev Cancer 2: 442-454, 2002.

8. Shook D and Keller R: Mechanisms, mechanics and function of epithelial-mesenchymal transitions in early development. Mech Dev 120: 1351-1383, 2003.

9. Lee MJ, Dedhar S, Kalluri R and Thompson EW: The epithelialmesenchymal transition: new insights in signaling, development, and disease. J Cell Biol 172: 973-981, 2006.

10. Ornitz DM, Xu J, Colvin JS, et al: Receptor specificity of the FGF family. J Biol Chem 271: 15292-15297, 1996.

11. Zhang X, Ibrahimi OA, Olsen SK, et al: Receptor specificity of the FGF family. J Biol Chem 281: 15694-15700, 2006.

12. Katoh M: WNT and FGF gene clusters. Int J Oncol 21: 1269-1273, 2002.

13. Katoh M and Katoh M: Evolutionary conservation of CCND1ORAOV1-FGF19-FGF4 locus from zebrafish to human. Int J Mol Med 12: 45-50, 2003.

14. Popovici C, Roubin R, Coulier F and Birnbaum D: An evolutionary history of the FGF superfamily. Bioessays 27: 849-857, 2005.

15. Katoh Y and Katoh M: Comparative genomics on FGF7, FGF10, $F G F 22$ orthologs, and identification of $f g f 25$. Int J Mol Med 16: 767-770, 2005

16. Finch PW, Rubin JS, Miki T, et al: Human KGF is FGF-related with properties of a paracrine effector of epithelial cells. Science 145: 752-755, 1989.

17. Boismenu R and Havran WL: Modulation of epithelial cell growth by intraepithelial $\gamma \delta \mathrm{T}$ cells. Science 266: 1253-1255, 1994.

18. auf demKeller U, Krampert M, Kümin A, Braun S and Werner S: Keratinocyte growth factor: effects on keratinocytes and mechanisms of action. Eur J Cell Biol 83: 607-612, 2004.

19. Arman E, Haffner-Krausz R, Gorivodsky M and Lonai P: Fgfr2 is required for limb outgrowth and lung-branching morphogenesis. Proc Natl Acad Sci USA 96: 11895-11899, 1999.

20. Spencer-Dene B, Sala FG, Bellusci S, et al: Stomach development is dependent on FGF10/FGFR2b-mediated signaling. Gastroenterology 130: 1233-1244, 2006.

21. Umemori H, Linhoff MW, Ornitz DM and Sanes JR: FGF22 and its close relatives are presynaptic organizing molecules in the mammalian brain. Cell 118: 257-270, 2004.

22. Katoh M and Katoh M: WNT signaling pathway and stem cell signaling network. Clin Cancer Res 13: 4042-4045, 2007.

23. Katoh M: WNT signaling in stem cell biology and regenerative medicine. Curr Drug Targets 9: 565-570, 2008

24. Katoh M: Dysregulation of stem cell signaling network due to germline mutation, SNP, Helicobacter pylori infection, epigenetic change, and genetic alteration in gastric cancer. Cancer Biol Ther 6: 832-839, 2007.

25. Katoh M: Cancer genomics and genetics of FGFR2. Int J Oncol 33: 233-237, 2008.

26. Wilkie AOM: Bad bones, absent smell, selfish testes: the pleiotropic consequences of human FGF receptor mutations. Cytokine Growth Factor Rev 16: 187-203, 2005.

27. Pollock PM, Gartside MG, Dejeza LC, et al: Frequent activating FGFR2 mutations in endometrial carcinomas parallel germline mutations associated with craniosynostosis and skeletal dysplasia syndromes. Oncogene 26: 7158-7162, 2007.

28. Davies H, Hunter C, Smith R, et al: Somatic mutations of the protein kinase gene family in human lung cancer. Cancer Res 65: 7591-7595, 2005.

29. Jang JH, Shin KH and Park JG: Mutations in FGFR2 and FGFR3 genes associated with human gastric and colorectal cancers. Cancer Res 61: 3541-3543, 2001. 
30. Adnane J, Gaudray P, Dionne CA, et al: BEK and FLG are amplified in subsets of human breast cancers. Oncogene 6: 659-663, 1991

31. Katoh M and Terada M: Oncogenes and tumor suppressor genes. In: Gastric Cancer. Nishi M, et al (eds). Springer-Verlag, Tokyo, pp196-208, 1993.

32. Moffa AB, Tannheimer SL and Ethier SP: Transforming potential of alternatively spliced variants of FGFR2 in human mammary epithelial cells. Mol Cancer Res 2: 643-652, 2004.

33. Easton DF, Pooley KA, Dunning AM, et al: Genome-wide association study identifies novel breast cancer susceptibility loci. Nature 447: 1087-1093, 2007.

34. Hunter DJ, Kraft P, Jacobs KB, et al: A genome-wide association study identifies alleles in FGFR2 associated with risk of sporadic postmenopausal breast cancer. Nat Genet 39: 870-874, 2007.

35. Huijts PE, Vreeswijk MP, Kroeze-Jansema KH, et al: Clinical correlates of low-risk variants in FGFR2, TNRC9, MAP3KI, LSP1 and 8q24 in a Dutch cohort of incident breast cancer cases. Breast Cancer Res 9: R78, 2007.

36. Meyer KB, Maia A-T, O'Reilly M, et al: Allele-specific upregulation of $F G F R 2$ increases susceptibility to breast cancer. PLoS Biol 6: e108, 2008.

37. McGrath M, Lee IM, Buring J, Hunter DJ and De Vivo I: Novel breast cancer risk alleles and endometrial cancer risk. Int J Cancer 123: 2961-2964, 2008.

38. Gates MA, Tworoger SS, Terry KL, et al: Breast cancer susceptibility alleles and ovarian cancer risk in 2 study populations. Int J Cancer 124: 729-733, 2008.

39. Chaffer CL, Dopheide B, Savagner P, et al: Aberrant FGF receptor signaling in bladder and other cancers. Differentiation 75: 831-842, 2007

40. Katoh M: Epithelial-mesenchymal transition in gastric cancer. Int J Oncol 27: 1677-1683, 2005.

41. Katoh Y and Katoh M: Hedgehog signaling, epithelial-tomesenchymal transition, and miRNA. Int J Mol Med 22: 271-275, 2008.

42. Matsubara A, Kan M, Feng S and McKeehan WL: Inhibition of growth of malignant rat prostate tumor cells by restoration of FGFR2. Cancer Res 58: 1509-1514, 1998.

43. Ricol D, Cappellen D, El Marjou A, et al: Tumour suppressive properties of FGFR2b in human bladder cancer. Oncogene 18: 7234-7243, 1999.

44. Grose R, Fantl V, Werner S, et al: The role of Fgfr2b in skin homeostasis and cancer development. EMBO J 26: 1268-1278, 2007.

45. Jameson J, Ugarte $\mathrm{K}$, Chen $\mathrm{N}$, et al: A role for skin $\gamma \delta \mathrm{T}$ cells in wound repair. Science 296: 747-749, 2002

46. Girardi M, Oppenheim DE, Steele CR, et al: Regulation of cutaneous malignancy by $\gamma \delta$ T cells. Science 294: 605-609, 2001.

47. Tong KI, Kobayashi A, Katsuoka F and Yamamoto M: Two-site substrate recognition model for the Keap1-Nrf2 system: a hinge and latch mechanism. Biol Chem 387: 1311-1320, 2006.

48. Reardon W, Winter RM, Rutland P, et al: Mutations in the FGFR2 gene cause Crouzon syndrome. Nat Genet 8: 98-103, 1994

49. Jabs EW, Li X, Scott AF, et al: Jackson-Weiss and Crouzon syndromes are allelic with mutations in FGFR2. Nat Genet 8 : 275-279, 1994.

50. Passos-Bueno MR, Wilcox WR, Jabs EW, et al: Clinical spectrum of FGFR mutations. Hum Mutat 14: 115-125, 1999.

51. O'Donovan MC, Norton N, Williams H, et al: Analysis of 10 independent samples provides evidence for association between schizophrenia and a SNP flanking FGFR2. Mol Psychiatry (In press).

52. Nobel ME, Endicott JA and Johnson LN: Protein kinase inhibitors: insight into drug design from structure. Science 303: 1800-1805, 2004.
53. Garber K: The second wave in kinase cancer drugs. Nat Biotechnol 24: 127-130, 2006

54. Mohammadi M, Froum S, Hamby JM, et al: Crystal structure of an angiogenesis inhibitor bound to the FGF receptor tyrosine kinase domain. EMBO J 17: 5896-5904, 1998.

55. Koziczak M, Holbro T and Hynes NE: Blocking of FGFR signaling inhibits breast cancer cell proliferation through downregulation of D-type cyclins. Oncogene 23: 3501-3508 2004.

56. Dutt A, Salvesen HB, Chen TH, et al: Drug-sensitive FGFR2 mutations in endometrial carcinoma. Proc Natl Acad Sci USA 105: 8713-8717, 2008

57. Byron SA, Gartside MG, Wellens CL, et al: Inhibition of activated FGFR2 in endometrial cancer cells induces cell death despite PTEN abrogation. Cancer Res 68: 6902-6907, 2008.

58. Sun L, Tran N, Tang F, et al: Synthesis and biological evaluations of 3-substituted indolin-2-ones: a novel class of tyrosine kinase inhibitors that exhibit selectivity toward particular receptor tyrosine kinases. J Med Chem 41: 2588-2603, 1998.

59. Mohammadi M, McMahon G, Sun L, et al: Structures of the tyrosine kinase domain of fibroblast growth factor receptor in complex with inhibitors. Science 276: 955-960, 1997.

60. Wedge SR, Kendrew J, Hennequin LF, et al: AZD2171: a highly potent, orally bioavailable, VEGFR2 tyrosine kinase inhibitor for the treatment of cancer. Cancer Res 65: 4389-4400, 2005.

61. Nakamura K, Yashiro M, Matsuoka T, et al: A novel molecular targeting compound as FGFR2 phosphorylation inhibitor, Ki23057, for scirrhous gastric cancer. Gastroenterology 131: 1530-1541, 2006.

62. Katoh M: RNA technology targeted to the WNT signaling pathway. Cancer Biol Ther 7: 275-277, 2008.

63. Li S, Christensen C, Kiselyov VV, et al: Fibroblast growth factorderived peptides: functional agonists of the fibroblast growth factor receptor. J Neurochem 104: 667-682, 2008.

64. Ulrich H: DNA and RNA aptamers as modulators of protein function. Med Chem 1: 199-208, 2005.

65. Lee HK, Choi YS, Park YA and Jeong S: Modulation of oncogenic transcription and alternative splicing by beta-catenin and an RNA aptamer in colon cancer cells. Cancer Res 66: 10560-10566, 2006.

66. Ireson CR and Kelland LR: Discovery and development of anticancer aptamers. Mol Cancer Ther 5: 2957-2962, 2006.

67. Reichert JM, Rosensweig CJ, Faden LB and Dewitz MC: Monoclonal antibody successes in the clinic. Nat Biotechnol 23: 1073-1078, 2005.

68. Fire A, Xu S, Montgomery MK, et al: Potent and specific genetic interference by double-stranded RNA in Caenorhabditis elegans. Nature 391: 806-811, 1998.

69. Elbashir SM, Harborth J, Lendeckel W, et al: Duplexes of 21-nucleotide RNAs mediate RNA interference in cultured mammalian cells. Nature 411: 494-498, 2001.

70. Liang Z, Wu H, Reddy S, et al: Blockade of invasion and metastasis of breast cancer cells via targeting CXCR4 with an artificial microRNA. Biochem Biophys Res Commun 363: 542-546, 2007.

71. Katoh M: Demand for user-friendly 'omics' web service utilizing next-generation supercomputer in the sequencing era. Mol Cancer Genet 1: 1-2, 2008

72. Katoh $\mathrm{M}$ and Katoh M: Bioinformatics for cancer management in the post-genome era. Technol Cancer Res Treat 5: 169-176, 2006.

73. Collins I and Workman P: New approaches to molecular cancer therapeutics. Nat Chem Biol 12: 689-700, 2006. 